\title{
Uncharted Territory-Rehabilitation Potential in a Prolonged COVID-19 ICU Stay Complicated by PRES
}

\author{
Samuel Moody, Vinay Parambil, Fayez Morcos \\ Floyd Neurorehabilitation Unit, Northern Care Alliance NHS Trust, Rochdale, UK \\ Email: Samuel.moody@doctors.org.uk
}

How to cite this paper: Moody, S., Parambil, V. and Morcos, F. (2021) Uncharted Territory-Rehabilitation Potential in a Prolonged COVID-19 ICU Stay Complicated by PRES. Open Access Library Journal, 8: e7627.

https://doi.org/10.4236/oalib.1107627

Received: June 10, 2021

Accepted: August 22, 2021

Published: August 25, 2021

Copyright $\odot 2021$ by author(s) and Open Access Library Inc.

This work is licensed under the Creative Commons Attribution International License (CC BY 4.0).

http://creativecommons.org/licenses/by/4.0/

\begin{abstract}
The long-term complications of COVID-19 and its management, and rehabilitation potential post-recovery remain poorly understood. Here we describe the case of posterior reversible encephalopathy syndrome and subsequent neuro-rehabilitation outcome of a patient following an extended ICU stay with COVID-19 infection (and complications). Rehabilitation potential and mirroring research are discussed.
\end{abstract}

\section{Subject Areas}

COVID-19, Neurorehabilitation

\section{Keywords}

COVID-19, Neurorehabilitation, Posterior Reversible Encephalopathy Syndrome

\section{Introduction}

While COVID-19 (CV-19) has presented new challenges for almost all medical specialties, only recently have we begun to appreciate its neuro-rehabilitation sequelae and outcomes-both disease process and our approach to treatment. With a paucity of documented sequelae, and faced with the challenge of an ever-increasing patient burden, it is important to work towards building a record of patient outcomes to guide our future management. Here we describe the effects of a prolonged period of intubation and ventilation due to CV-19 pneumonitis, and the outcome following a period of intensive neurorehabilitation at a dedicated facility. There is an increasing library of research detailing the relationship between CV-19, PRES, and PRES-like syndromes, but little on the neu- 
ropsychiatric sequelae of CV-19 infection. With this paper, we hope to expand our understanding of the neurological sequelae of CV-19 infection.

\section{Admission}

\subsection{Acute Admission}

A previously well, 57-year-old male taxi driver was admitted via the emergency department with a one-day history or worsening shortness of breath. He had a past history of medically managed hypertension, hypercholesterolemia and previous $\mathrm{L}$ occipital infarct (from which he made a full recovery). A COVID-19 $(\mathrm{CV}-19)$ polymerase chain reaction (PCR) swab taken on admission returned positive and he was started on doxycycline and hydrocortisone (as per local policy at the time). He continued to deteriorate in hospital and was admitted to the Intensive Care Unit (ICU) after developing type 1 respiratory failure, where he was intubated and ventilated and commenced on Lopinavir/Ritonavir and methylprednisolone. His ICU stay was complicated by a prolonged lung abscess requiring IV antibiotics.

He was weaned off ventilatory support on day 90 and stepped down. Unfortunately, he became acutely confused and following a prolonged seizure was readmitted to ICU and re-intubated; a CT head showed new subcortical hypodensities (in addition to the established left occipital infarct) and a radiological diagnosis of Posterior Reversible Encephalopathy Syndrome (PRES) was made. Following aggressive blood pressure and seizure management he was re-extubated five days later; he was admitted to the Floyd Neurorehabilitation unit once medically optimized.

\subsection{Admission to the Neurorehabilitation Unit}

On admission he was notably frail, with an unsafe swallow (managed with thickened fluids). His activity was limited to a tilt-in-space wheelchair for maximum four hours per day. Glasgow Coma Score was 15/15 and abbreviated mental test score was 9/10. Cranial nerves were normal except for an isolated right XII nerve palsy with right-sided tongue deviation. There was significant wasting of the intrinsic, thenar and hypothenar muscles bilaterally; reflexes were globally diminished with normal sensation and Medical Research Council (MRC) muscle scale power III-IV throughout. Systolic hypertension was also noted (Figure 1).

\subsection{Clinical Course and Discharge}

A remarkable functional improvement was seen during the patient's stay at the Rehabilitation Unit. With consistent and targeted therapy, he regained strength and his swallow improved to thin fluids and normal diet. He slowly regained his mobility and walked independently from the unit on discharge. His cognition also improved over time, returning to normal (normal cognition, sequential and memory task functionality). He was troubled by globus pharyngeus and vomiting throughout his stay; video fluoroscopy was normal and barium swallow detected 


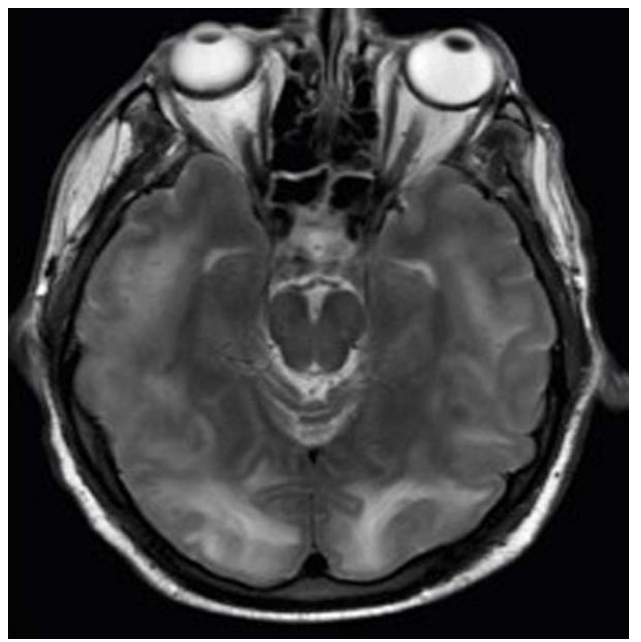

Figure 1. MRI brain. Note the high occipital signal return.

trace silent aspiration, for which he is still being investigated to delineate the cause.

He did note some psychiatric disturbance pertaining to his experience, including visualizing his stay in ICU and hearing the medical staff there. He was treated with sertraline, with benefit; however, on discharge he was still suffering regular nightmares about his ICU stay.

Aggressive control of his blood pressure was maintained on the unit to ensure resolution of his PRES and he was discharged with anti-epileptics.

\section{Discussion}

This gentleman, with a background of cerebral infarct and hypertension, had a much-prolonged ITU stay of 95 days due to CV-19 pneumonitis complicated by seizures, pneumonia and lung abscess. Based on his seizure activity, imaging and abnormal blood pressure her received a diagnosis of PRES towards the end of his ITU stay, further complicating his route to recovery.

We use this case to illustrate firstly the remarkable outcome of a gentleman who was extremely unwell on admission to us; on discharge he was fully mobile and capable of independent living. Secondly, it is a caution to rehabilitation specialists of the neurological sequelae following CV-19 related insult (in this case, PRES) - these issues continue to travel with the patient after initial recovery and require ongoing careful control in the rehabilitation environment (and beyond). It is also worth noting that there is some evidence linking high-dose steroid therapy and the subsequent occurrence of PRES [1], leaving clinicians with a treatment dilemma.

\section{The Post-CV-19 Brain and Its Relationship with PRES}

CV-19 survivors have been left with notable neurological symptoms such as dizziness, headache and impaired consciousness [2]. More serious sequelae (including encephalitis, stroke, micro-and macro-haemorrhage, encephalopathy and cerebral venous embolism) have also been described [3], and although more in- 
trospection is required the bulk of neurological injury is secondary to inflammatory or immune-mediated injury [4]. Angiotensin-converting enzyme is also being implicated in the rapid vasogenic oedema in brain changes seen with CV-19 [5], and a link between this and PRES could be postulated-though difficult to elucidate, as PRES can also be responsible for similar neurological symptoms [6]. An increasing number of cases of PRES and CV-19 are being described in literature [7] [8], as are CV-19 and 'PRES-like' syndromes, including cortical blindness, leukoencephalopathies and carotid thrombosis [9] [10]. We also note that a case of isolated unilateral CNXII nerve palsy has been reported in a case study from Portugal [11], mirroring our experience here. Treatment for PRES focuses on aggressive blood pressure and seizure control, aims that would be shared by clinicians managing CV-19 complications [12].

So far, little research exists describing long-term brain changes after CV-19 infection in its entirety. The neuropsychiatric complications of COVID, as seen here with the gentleman's recurrent nightmares, also suffer from a paucity of research and will require attentive follow-up in the post-CV-19 rehabilitation landscape.

There is no consistent, standardised data on how many critical care patients need-or go on to receive-specialist inpatient rehabilitation, and the burden on rehabilitation services from post-COVID patients remains unknown [13]. A deeper understanding of the lasting neurological effects of CV-19 will be critical for service planning and delivery in the coming years and, as shown, patients have the capacity to benefit greatly from targeted and extended rehabilitation input.

\section{Conclusion}

We have described a complex case of CV-19 requiring a prolonged ITU stay, complicated by PRES, before making a good recovery in a dedicated rehabilitation setting. This case joins a number of other publications connecting PRES and PRES-like syndromes to CV-19 infection, but we note a paucity of research around long-term biological and neuropsychiatric complications of severe CV-19 infection. Patients such as this would benefit from a better understanding of CV-19 infection and its effect on neuro-rehabilitation potential, and such knowledge could be used in the future to tailor rehabilitation care towards the particular challenges $\mathrm{CV}-19$ presents.

\section{Acknowledgements}

We send many thanks to the neurorehabilitation team at the Floyd Neurorehabilitation Unit, Rochdale, for their exemplary work.

\section{Conflicts of Interest}

The authors declare no conflicts of interest.

\section{References}

[1] Sukumaran, S., et al. (2010) Posterior Reversible Encephalopathy Syndrome as a 
Consequence of High-Dose Steroid Administration after Autologous PBSCT. Bone Marrow Transplantation, 45, 779-780. https://doi.org/10.1038/bmt.2009.211

[2] Varatharai, A., et al. (2020) Neurological and Neuropsychiatric Complications of COVID-19 in 153 Patients: A UK-Wide Surveillance Study. Lancet Psychiatry, 7, 875-882. https://doi.org/10.1016/S2215-0366(20)30287-X

[3] Paterson, R.W., et al. (2020) The Emerging Spectrum of COVID-19 Neurology: Clinical, Radiological and Laboratory Findings. Brain, 143, 3104-3120. https://doi.org/10.1093/brain/awaa240

[4] Sheraton, M., Deo, N., Kashyap, R. and Surani, S. (2020) A Review of Neurological Complications of COVID-19. Cureus, 12, e8192.

https://doi.org/10.7759/cureus.8192

[5] Saavedra, J.M. (2020) COVID-19, Angiotensin Receptor Blockers, and the Brain. Cellular and Molecular Neurobiology, 40, 667-674.

https://doi.org/10.1007/s10571-020-00861-y

[6] Servillo, G., et al. (2003) Posterior Reversible Encephalopathy Syndrome (PRES) in Critically Ill Obstetric Patients. Intensive Care Medicine, 29, 2323-2326. https://doi.org/10.1007/s00134-003-1901-1

[7] Princiotta Cariddi, L., et al. (2020) Reversible Encephalopathy Syndrome (PRES) in a COVID-19 Patient. Journal of Neurology, 267, 3157-3160. https://doi.org/10.1007/s00415-020-10001-7

[8] Lallana, S., et al. (2021) Posterior Reversible Encephalopathy Syndrome (PRES) Associated with COVID-19. Clinical Neuroscience, 88, 108-112. https://doi.org/10.1016/j.jocn.2021.03.028

[9] Doo, F., et al. (2021) Rare presentations of COVID-19: PRES-Like Leukoencepalopathy and Carotid Thrombosis. Clinical Imaging, 69, 94-101. https://doi.org/10.1016/j.clinimag.2020.07.007

[10] Kaya, Y., Kara, S., Akinci, C. \& Kocaman, A.S. (2020) Transient Cortical Blindness in COVID-19 Pneumonia; A Pres-Like Syndrome: Case Report. Neurological Sciences, 413, 116858. https://doi.org/10.1016/j.jns.2020.116858

[11] Costa Martins, D., Branco Ribiero, S., Jesus Pereira, I., Mestre, S. and Rios, J. (2020) Unilateral Hypoglossal Nerve Palsy as a COVID-19 Sequel. American Journal of Physical Medicine \& Rehabilitation, 99, 1096-1098. https://doi.org/10.1097/PHM.0000000000001607

[12] Striano, P., et al. (2005) Clinical Spectrum and Critical Care Management of Posterior Reversible Encepalopathy Syndrome (PRES). Medical Science Monitor, 11, 549-553.

[13] Thornton, J. (2020) COVID-19: The Challenge of Patient Rehabilitation after Intensive Care. BMJ, 369, Ariticle No. m1787. https://doi.org/10.1136/bmj.m1787 